\title{
Use of erbium as a burnable absorber for the VVER reactor core life extension*
}

\author{
Saleh H. Alassaf ${ }^{1}$, Vladimir I. Savander ${ }^{1}$, Ahmed A. Hassan ${ }^{1}$ \\ 1 National Research Nuclear University "MEPhI”, 31 Kashirskoye shosse, Moscow, 115409, Russia \\ Corresponding author: Vladimir I. Savander (VISavander@mephi.ru)
}

Academic editor: Georgy Tikhomirov • Received 12 April 2020 Accepted 10 Sepember 2020 • Published 20 November 2020

Citation: Alassaf SH, Savander VI, Hassan AA (2020) Use of erbium as a burnable absorber for the VVER reactor core life extension. Nuclear Energy and Technology 6(4): 275-279. https://doi.org/10.3897/nucet.6.60563

\begin{abstract}
The paper presents the results of a computational and theoretical analysis concerned with the use of erbium as a burnable absorber in VVER-type reactors. Partial refueling options for the reactor life extension to 18 and 24 months is considered, the refueling ratio being equal to three for the 18-month life and to two for the 24-month life. Erbium is expected to be present in all fuel elements in the FA with the same weight content. The influence of the erbium weight content on such neutronic characteristics of the reactor and fuel as burn-up, reactivity coefficients, residual volume of "liquid" control, and amounts of the liquid radioactive waste (LRW) formed was assessed.

The calculations were performed using a simplified model of refueling without FA reshuffling. An infinite array of polycells consisting of FAs with different in-core times was considered. The escape of neutrons from the core was taken into account by selecting the critical value $K_{\infty}$ at the end of life.

Erbium does not burn up in full for the lifetime which affects the fuel burn-up as compared with the liquid excessive reactivity compensation system. The reduction is $0.7 \%$ per $0.1 \%$ of the erbium weight load in the fuel elements. This, however, also reduces the maximum content of the boron absorber in the coolant and the LRW accumulation in the ratio of $5 \%$ per $0.1 \%$ of the erbium weight load.

Erbium influences the spectral component of the coolant temperature reactivity coefficient which turns out to be negative even with its minor weight fraction in fuel elements, and a reduction in the boron absorber fraction leads to a positive value of the density reactivity coefficient. As a result, the overall coolant temperature reactivity coefficient has a negative value throughout the lifetime.
\end{abstract}

\section{Keywords}

VVER, burnable absorber, erbium, reactivity margin, GETERA, liquid excessive reactivity compensation system, liquid radioactive waste (LRW)

\section{Introduction}

A core life extension in reactors with partial refueling leads to an increased capacity utilization factor (CUF) and reduced unit costs of nuclear hazardous refueling operations which, combined, improves the economic efficiency of the NPP. However, an extended core life causes a proportional growth in the fuel reactivity margin that requires

* Russian text published: Izvestiya vuzov. Yadernaya Energetika (ISSN 0204-3327), 2020, n. 3, pp. 62-71.

Copyright Alassaf SH et al. This is an open access article distributed under the terms of the Creative Commons Attribution License (CC-BY 4.0), which permits unrestricted use, distribution, and reproduction in any medium, provided the original author and source are credited. 
to be compensated throughout the reactor lifetime. To this end, along with the liquid system based on dissolution of boron absorber in the coolant, reactors of the VVER-1000 type use a gadolinium-based burnable absorber which is integrated into the fuel. With a high reactivity margin, if liquid control alone is used, the density reactivity coefficient can become negative, and the coolant temperature reactivity can become positive. Therefore, it is required to reduce the maximum concentration of the boron absorber in the coolant by increasing the share of the reactivity margin compensated through the use of burnable absorbers.

Natural gadolinium, a strong absorber of thermal neutrons, is used most extensively in Russian reactors (DOE Fundamentals Handbook 1993). It is introduced into a small number of (gadolinium) fuel elements but with a relatively high concentration which blocks efficiently the thermal neutron flux in gadolinium fuel elements. The concentration of gadolinium is selected proceeding from a condition that it should burn up in full in the makeup fuel for the initial irradiation cycle. In this case, it does not affect the average burnup of the unloaded fuel which could be obtained in the event of a purely liquid system for the excessive reactivity compensation. In connection with the fact that gadolinium fuel elements reduce the thermal neutron flux in adjoining fuel elements, FAs will have highly non-uniform power density fields occurring in them. Besides, the fuel enrichment in gadolinium fuel elements is smaller than in other fuel elements, which, with the number of gadolinium rods being large, leads to less uranium loaded into the core. It is appropriate to note that the presence of gadolinium in gadolinium fuel elements reduces the fuel thermal conductivity.

There are no these drawbacks in the event of using relatively weak absorbers which can be introduced either into all fuel elements (Frybort 2012) or into many of them, this being equivalent to using a homogeneous absorber in the entire FA. And small concentrations of such absorber in fuel elements do not require reduced enrichment and do not affect the thermal conductivity of fuel. Natural erbium is used for such absorber in Russia. The use of erbium eliminates the problem of ensuring nuclear safety during fabrication and handling of fresh fuel with its enrichment increased to above 5\% (Fedosov 2018). A feature of erbium as a thermal neutron absorber is that the dependence of its absorption micro-cross-section in this region of energies has a resonance at the energy $E=0.41 \mathrm{eV}$ (Franceschini Fausto and Petrović Bojan 2009). This feature leads to the spectral component of the overall coolant temperature reactivity coefficient having negative values which provides for a negative value of the overall temperature coefficient as well even with relatively small quantities of erbium in fuel elements. The thermal conductivity of fuel does not practically change when erbium is used in fuel elements. However, erbium isotopes do not have enough time for burning up for the core lifetime (Franceschini Fausto 2017), this resulting in the fuel burn-up being dependent on the content of erbium in fuel elements.
At the present time, reactors of the VVER-1000 and VVER-1200 types have an 18-month life with a length of 480 days and a further extension to 510 to 540 days (Semchenkov 2014). With the makeup fuel enrichment of up to $4.95 \%$, such lifetime is achieved by using mixed refueling which suggests that some of the FAs operate in the core for three cycles, and the other operate for only two. This increases substantially the annual consumption of natural uranium. The same will take place if a 24-month life is introduced but this will however lead to an increase in the burn-up reactivity margin. To reduce the natural uranium consumption, refueling requires to be multiple with all FAs remaining in the core for an equal number of cycle. This is achieved by increasing the initial enrichment of the makeup fuel to above the level of 5\% (Savander and Al'assaf 2019). The paper considers exactly this option with the refueling ratio being equal to three for the 18-month life and to two for the 24-month life.

\section{Computational analysis}

As part of a simplified fuel burn-up model for a partial core refueling option, it has been assessed how the quantity of erbium absorber introduced as the burnable absorber into all fuel elements in the FA influences the unloaded fuel burn-up, the coolant density and temperature reactivity coefficients, and the volume of the liquid compensation system. The major simplification consists in that refueling is considered without FA reshuffling in the core.

Computational studies were performed using the GETERA code (Pryanichnikov 2009), which makes it possible to determine the spatial and energy distributions of neutrons in elementary cells and in selected periodic fragments of the core based on the first collision probability method, as well as the change of neutron multiplication factor and the isotopic composition of fuel in the process of burn-up with refueling being partial.

The calculation model was based on identifying the periodic array of polycells consisting of FAs with different irradiation times in the core. Partial refueling is considered without reshuffling (Pryanichnikov 2009) with average values of the coolant and fuel temperature.

Primarily, a problem was considered concerning the assessment of how the weight fraction of erbium in fuel elements influences the burn-up of unloaded fuel (Bergelson et al. 2011). The weight content of erbium dioxide in fuel elements varied in a range of 0.3 to $1.1 \%$ with an interval of 0.2 wt. \%. An option with a liquid system for the excessive reactivity compensation without using burnable absorbers was taken as the reference one. Further, the unloaded fuel burn-up was calculated for all options with erbium fuel and the relative loss in burn-up was computed relative to the reference option $\left(B_{0}-B\left(x_{\mathrm{Er}}\right)\right) / B_{0}$, where $B_{0}$ is the burn-up of fuel without erbium, and $x_{\mathrm{Er}}$ is the weight content of erbium in fuel elements. The calculation results are presented in Fig. 1. 


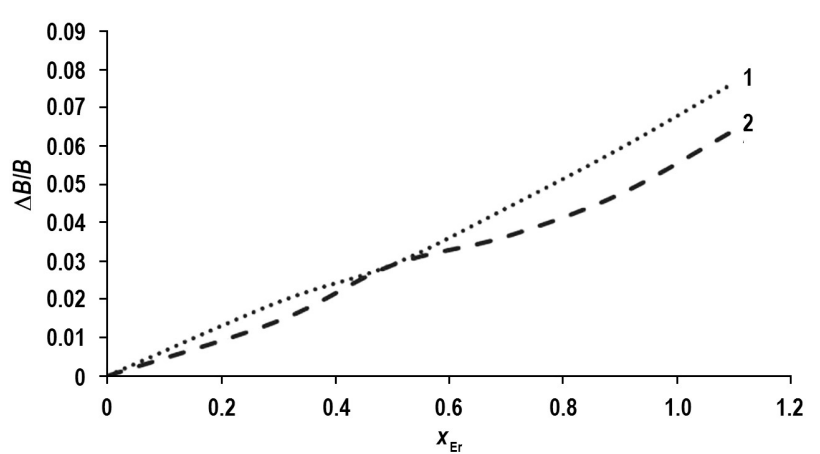

Figure 1. Relative loss in the fuel burn-up as a function of the erbium content in fuel: 1-18-month life; 2-24-month life.

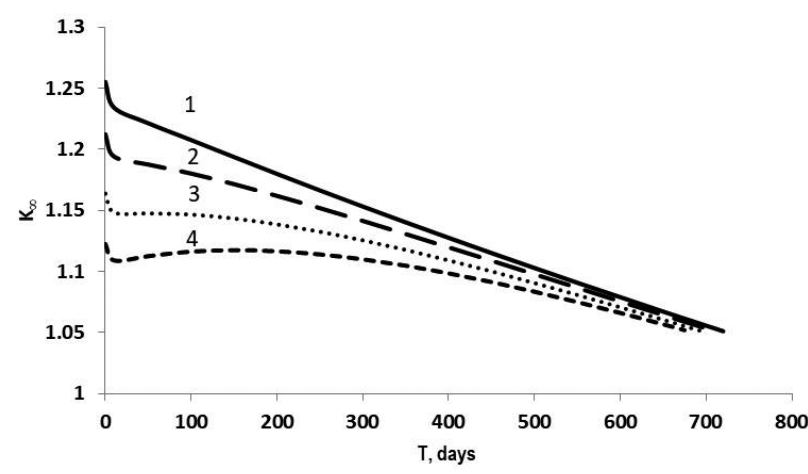

Figure 2. Polycell average neutron multiplication factor as a function of time with different erbium loads in fuel elements for the refueling ratio of two: $1-\operatorname{Er} 0 \% ; 2-\operatorname{Er} 0.3 \% ; 3-\operatorname{Er} 0.7 \% ; 4-1.1 \%$.

With loads of erbium dioxide in fuel elements being relatively small (up to $1 \%$ ), the dependence of the loss in burn-up on the content of erbium in fuel elements is linear and much smaller than the loss during the transition from a 12-month life to an 18-month or 24-month life (10 to 20\%).

We shall consider the influence of the erbium absorber on the overall coolant temperature reactivity coefficient. It is known to consist of two terms

$$
\frac{d \rho}{d T_{t h}}=\frac{d \rho}{d T_{t h}}\left(\gamma_{t h}=\mathrm{const}\right)+\frac{d \rho}{d \gamma_{t h}}\left(T_{t h}=\mathrm{const}\right) \frac{d \gamma_{t h}}{d T_{t h}}
$$

where $r$ is reactivity, $T_{\text {th }}$ is the coolant temperature, and $\gamma_{\text {th }}$ is the coolant density.

The first term we shall designate $\alpha_{\mathrm{m}}$ is the spectral component which is connected with the neutron gas temperature variation in response to the moderator temperature variation. For non-fissile isotopes, the dependence of the radiation capture micro-cross-section on the energy in the thermal region changes in accordance with the $1 / V$ law such that the thermal spectrum average micro-cross-section will decrease as the moderator temperature increases. Fissionable isotopes have a resonance in the spectrum's thermal region $(E=0.3 \mathrm{eV})$, which results in the averaged radiation capture and fission cross-sections growing with the moderator temperature increase. This reactivity coefficient will be positive in the absence of erbium. However,

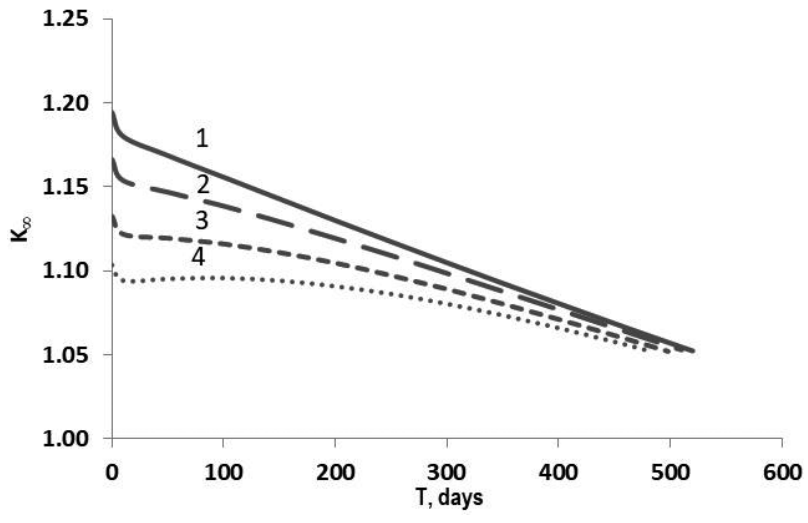

Figure 3. Polycell average neutron multiplication factor as a function of time with different erbium loads in fuel elements for the refueling ratio of three: $1-\operatorname{Er} 0 \% ; 2-\operatorname{Er} 0.3 \% ; 3-\operatorname{Er} 0.7 \% ; 4-1.1 \%$.

since even erbium isotopes also have a resonance at the energy $E=0.41 \mathrm{eV}$, the cumulative effect can be negative. So this coefficient depends largely on the concentration of erbium in fuel. Therefore, this term will have a negative sign if there is a certain quantity of erbium in fuel.

The second term designated $\mathrm{a}_{\mathrm{g}}$ is the density reactivity coefficient which, with a liquid system used for the excessive reactivity compensation, is defined to a great extent by the content of boron in the coolant. The higher is the concentration of boron in the coolant, the smaller is the value of the density reactivity coefficient. The calculation has shown that this value proved to be negative at the beginning of a regular life in the event of a purely liquid excessive reactivity compensation system for a 24-month life. For this case, as a result, the overall temperature reactivity coefficient turned out to be positive.

Since a simplified partial refueling model is used in the study, then relative reactivity coefficient values are presented for the computational analysis. Temperature reactivity coefficient and its components, which meet the end reactor state prior to refueling with only a liquid system employed for the excessive reactivity compensation, are used as normalizing values. In this state, the concentration of the boron absorber is equal to zero, and the reactivity coefficients are defined only by the water-fuel ratio value which is equal for all considered options. In particular, the values of these coefficients do not depend on the refueling ratio.

The largest concentration of the boron absorber in the core is normally achieved at the beginning of a regular life. This value was calculated for all options under consideration. Figs 2 and 3 present the variation in the polycell neutron multiplication factor on time throughout the cycle with different erbium loads in the makeup fuel from which it can be seen that there is no major reactivity excursion during erbium burn-up.

Such boron absorber concentration was added into the coolant for the calculation of reactivity coefficients as ensures the polycell criticality at the initial time. After that, the spectral and density components of the overall temperature reactivity coefficient for all considered life du- 


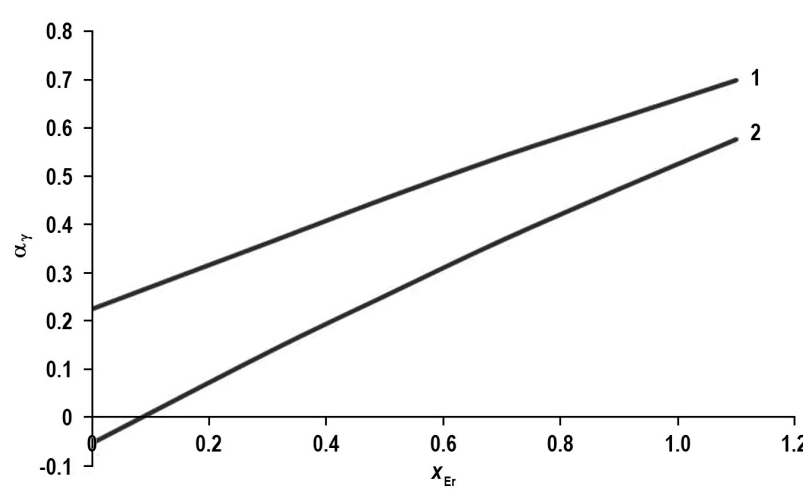

Figure 4. Relative value of the density reactivity coefficient as a function of the erbium load in fuel elements: 1-18-month life, 2-24-month life.

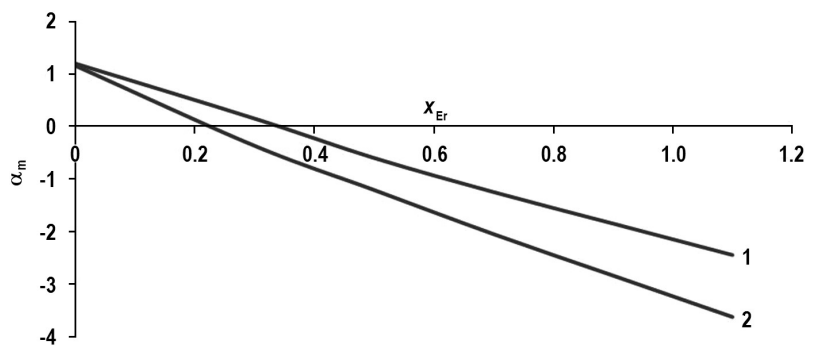

Figure 5. Relative value of the temperature reactivity coefficient's spectral component as a function of the erbium load in fuel: 1-18-month life; 2-24-month life.

ration and fuel erbium load options were determined by simply varying the coolant temperature and density. The relative calculated values are presented in Figs 4-6.

The results of the computational studies agree with the conclusion that a longer life and a larger initial concentration of boron in the coolant affect adversely the density reactivity coefficient reducing it to negative values for a 24-month life. When erbium is used, the initial concentration of boron in the coolant decreases and the density reactivity coefficient grows linearly reaching substantially positive values (up to 50 to $70 \%$ of its value in a purely uranium-water system) which meets the EOL core composition (Abu Sondos et al. 2019).

The spectral component of the reactivity coefficient ensures that negative values are achieved already with a small content of erbium in fuel elements and grows linearly in modulus with the growth in the erbium load in fuel, as it increases in modulus by a factor of 2.5 to 3 , this effect being dependent on the life duration.

It is natural that the overall coolant-moderator temperature reactivity coefficient also grows in modulus in a linear manner with the increase in the erbium load in fuel elements as it remains negative.

A major drawback of a liquid excessive reactivity compensation system is large amounts of low-level LRW accumulated at the NPP as the result of the coolant dilution for keeping the reactor critical. A comprehensive reduction in this LRW amount is a vital task involved in the VVER reactor improvement (Semchenkov 2014). In principle, the higher is the concentration of the erbium

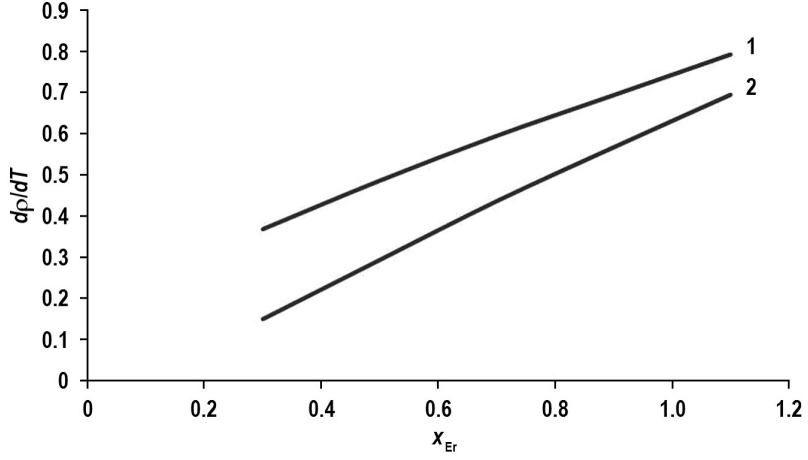

Figure 6. Relative value of the overall coolant temperature refuel: 1-18-month life; 2-24-month life.

absorber in fuel elements, the lower is the initial boron absorber concentration, and, therefore, the smaller is the LRW amount.

The physical peculiarity of the liquid reactivity margin regulation system is that the absorber is dissolved in the coolant and the coolant circulates in the primary circuit. As a result, the boron absorber is outside the core for much of the time. Therefore, the burn-up rate for this absorber is much lower than the fuel burn-up rate in the core, and regulation consists in reducing the quantity of the boron absorber in the coolant by diluting it with distilled coolant.

To estimate the LRW amount reduction, the lifetime boron concentration in the coolant required for the criticality was calculated. The LRW amount can be estimated based on this data since the LRW quantity is equal to the quantity of the distillate introduced into the circuit. We shall assume that the concentration of boron in the coolant was $C_{\mathrm{B}}(t)$ at the time $t$, and is expected to be $C_{\mathrm{B}}(t$ $+\Delta t$ ) e by the time $t+\Delta t$. And the quantity of the boron absorber to be removed from the circuit will be equal to $\Delta M_{\mathrm{B}}=\left(C_{\mathrm{B}}(t)-C_{\mathrm{B}}(t+\Delta t)\right) V_{\mathrm{K}}$, where $V_{\mathrm{K}}$ is the amount of the coolant in the primary circuit. This requires the amount $\Delta V(t)$, which is determined from the equality $C_{\mathrm{B}}(t) \cdot \Delta V(t)=\Delta M_{\mathrm{B}}$, to be taken from the circuit with boron. The same quantity of distillate needs to be added into the circuit to replace the separated coolant quantity. By equating these values, we shall have the equation for the LRW amount determination

$$
\frac{d V_{\mathrm{LRW}}}{V_{\mathrm{K}}}=\frac{d C_{\mathrm{B}}(t)}{C_{\mathrm{B}}(t)}
$$

the solution of which looks like

$$
V_{\mathrm{LRW}}=V_{\mathrm{K}} \ln \left(\frac{C_{\mathrm{B}}(0)}{C_{\mathrm{B}}(T)}\right)
$$

where $V_{\mathrm{LRW}}$ is the total lifetime accumulated quantity of LRW, and $C_{\mathrm{B}}(T)$ is the end concentration of boron at the boron control termination time.

The calculation results depend greatly on the assumed value of the residual boron absorber concentration in the 
coolant. This study uses the quantity of the LRW formed in the reactor with no burnable absorber, that is, for the case of purely boron control relative to the circuit volume, namely $n=V_{\mathrm{LRW}} / V_{\mathrm{K}}$. Given the value of this magnitude, we shall determine the final boron absorber concentration $C_{\mathrm{B}}(T)$ and shall further use this value for all options of the erbium load in fuel elements. This results in the equation

$$
V_{\mathrm{LRW}}\left(x_{\mathrm{Er}}\right)=V_{\mathrm{LRW}}(0)+V_{\mathrm{K}} \ln \left(\frac{C_{\mathrm{B}}\left(x_{\mathrm{Er}}, t=0\right)}{C_{\mathrm{B}}(0, t=0)}\right)
$$

from which we obtain the value of the relative LRW amount reduction

$$
\delta_{\mathrm{LRW}}\left(x_{\mathrm{Er}}\right)=\frac{V_{\mathrm{LRW}}(0)-V_{\mathrm{LRW}}\left(x_{\mathrm{Er}}\right)}{V_{\mathrm{LRW}}(0)}=\frac{1}{n} \ln \left(\frac{C_{\mathrm{B}}(0, t=0)}{C_{\mathrm{B}}\left(x_{\mathrm{Er}}, t=0\right)}\right)
$$

depending on the concentration of erbium in fuel elements (Fig. 7).

Since the fuel enrichment values for both partial refueling cases turned out to be close, the dependences of the relative reduction practically coincided as well, this being shown in Fig. 7. Assuming that the final concentration of boron for control is 10 times as low as the initial one, which is in line with the case $V_{\mathrm{LRW}} / V_{\mathrm{K}}=2$, then the LRW amount reduction change from 10 to $50 \%$ with the erbium load in fuel elements being in the above range.

\section{Conclusion}

The results of the computational studies for the applicability of erbium as burnable absorber for the VVER-type reactor life extension have been obtained using a simplified

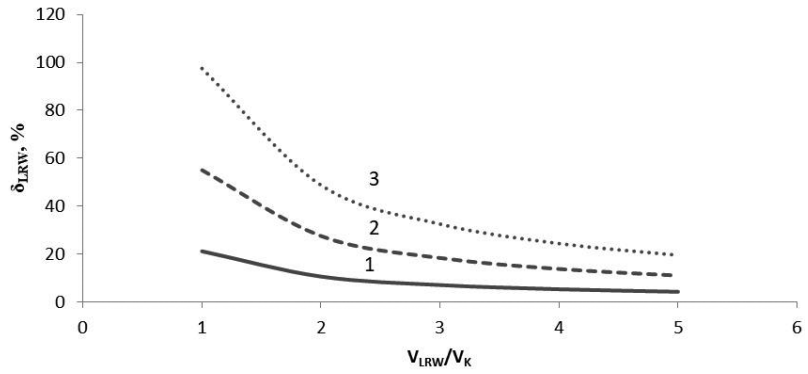

Figure 7. Relative LRW amount reduction as a function of the erbium concentration in fuel elements: $1-\operatorname{Er} 0.3 \% ; 2-\operatorname{Er} 0.7 \%$; $3-\operatorname{Er} 1.1 \%$.

model of partial refueling without the FAs reshuffled in the core. Options were considered with the core life extended to 18 to 24 months but with an integer refueling ratio which required an increased initial enrichment of the makeup fuel. Calculations have shown that varying the quantity of the erbium loaded into the fuel elements makes it possible to control the coolant-moderator temperature reactivity coefficient and ensures inherent safety properties (negative values of temperature reactivity coefficients).

It has been shown using a simple water exchange model for the boron absorber critical concentration control that the use of erbium as a burnable absorber makes it possible to reduce the LRW amount to $50 \%$. An increase in the erbium load in fuel elements reduces the burn-up of unloaded fuel by about $0.7 \%$ with the erbium load in fuel elements increased by 0.1 of wt. $\%$ as the reduction of the LRW amount in this case is about $5 \%$. A reduction in the fuel burn-up with the use of erbium leads to a decrease non uniformity factor of radial heat distribution in FA. However, the loss in burn-up can be reduced through an increase in the FA loading into the core's central region with the makeup fuel (Hashlamun et al. 2018).

\section{References}

- Abu Sondos MA, Demin VM, Savander VI (2019) Reducing the volume of the reactivity margin boron control when using a burnable absorber based on $\mathrm{GD}_{2} \mathrm{O}_{3}$ in the VVER-1200 reactor fuel. Globalnaya yadernaya bezopasnost 3(32): 56-65. https://doi.org/10.26583/gns2019-03-06 [in Russian]

- Bergelson BR, Belonog VV, Gerasimov AS, Tikhomirov GV (2011) VVER Nuclear Fuel Burnup with Different Absorbers. Atomic Energy 109(4): 240-245. https://doi.org/10.1007/s10512-011-9351-2

- DOE Fundamentals Handbook (1993) DOE Fundamentals Handbook: Nuclear Physics and Reactor Theory. U.S. Department of Energy. Vol. 2. Washington, 128 pp.

- Fedosov AM (2018) RBMK uranium-erbium fuel. Atomic Energy, 124(4): 221-226. https://doi.org/10.1007/s10512-018-0401-x

- Fausto F (2017) Use of isotopically modified erbium to improve fuel cycle economics in IRIS. In: Proceedings of the $5^{\text {th }}$ International Conference on Nuclear Option in Countries with Small and Medium Electricity Grids, INAC, 1-12.

- Fausto F, Bojan P (2009) Fuel with advanced burnable absorbers design for the IRIS reactor core: Combined Erbia and IFBA. An- nals of Nuclear Energy 36(8): 1201-1207. https://doi.org/10.1016/j. anucene.2009.04.005

- Frybort J (2012) Erbium burnable absorber for high-burn up fuels, WoS 000321966500252. In: Proceedings of the XIII ${ }^{\text {th }}$ International Scientific Conference EPE 2012. Czech Republic, 1299-1304.

- Hashlamun TM, Vygovskiy SB, Leskin ST, Duman AS (2018) Determination of the 18-month fuel cycle parameters for the fuel costs minimization based on FA designs in operation in VVER-1200 reactors. Izvestiya vuzov. Yadernaya energetika 3: 113-124. https://doi. org/10.26583/npe.2018.3.10 [in Russian]

- Pryanichnikov AV (2009) Description of the GETERA code. VANT. Ser. Fizika yadernykh reaktorov 3: 63-76. [in Russian]

- Savander VI, Al'assaf SH (2019) Analyzing the efficiency of using extended core life cycles in VVER reactor at foreign NPPs. Yadernaya fizika i inzhiniring 10(1): 5-8. [in Russian]

- Semchenkov Yu (2014) Use of fuel in VVER reactors: status and prospects. Rosenergoatom 11: 8-13. [in Russian] 